\title{
Integrating implementation science into covid-19 response and recovery
}

\author{
Lisa Hirschhorn professor ${ }^{12}$, Justin D Smith associate professor ${ }^{1}$, Miriam F Frisch research \\ associate $^{2}$, Agnes Binagwaho professor ${ }^{2}$
}

${ }^{1}$ Feinberg School of Medicine, Northwestern University, Chicago, IL, USA; ${ }^{2}$ University of Global Health Equity, Kigali Heights, Kigali, Rwanda

National and subnational responses to the covid-19 pandemic have varied in their implementation of critical evidence based interventions, including social distancing, handwashing, SARS-CoV-2 testing, and contact tracing. The variable success of such measures also reflects differences in the nature of initial outbreaks and contextual factors within communities and health systems. Implementation science methods can make all the difference, allowing us to build on these successes and ensure that new epidemiological and sociobehavioural models and other innovations can sustain and accelerate action to end this pandemic.

Implementation science focuses on applying rigorous frameworks, measures, and research designs, ${ }^{12}$ to study what, why, and how interventions are implemented in real world settings and tests strategies to improve their outcomes and impact.This helps policy makers, managers, and implementers understand existing and emerging evidence based interventions and choose strategies to implement them that take account of (and modify) contextual factors that influence success. Evidence produced through implementation research can inform countries' responses as epidemics emerge and as strategies are considered and enacted globally and locally for subsequent phases.

Integrating this approach requires strong diverse partnerships-including among implementers, researchers, policy makers, and communities-within and across countries. The rapid spread of covid-19 highlights the need to use implementation science methods from the outset of outbreaks. Evidence from the 2014 Ebola outbreak on how strategies were chosen to tackle the epidemic and mitigate the damage to essential health services was slow to emerge and limited in its dissemination. ${ }^{3}$ As a result, knowledge on what worked then and might be relevant in the covic-19 pandemic was lost. ${ }^{45}$

To respond effectively to covid-19, countries must identify, understand, and incorporate knowledge learnt throughout the course of the pandemic. Many countries are implementing established interventions. But they are not always applying the learning that implementation science can drive to select, analyse, and adapt data driven strategies for effective outcomes in different contexts and at different stages of the evolving pandemic. $^{6-8}$

\section{Implementation differences}

South Korea and the UK show this well, having implemented similar evidence based interventions for the covid-19 pandemic at different speeds and with implementation strategies that had different contexts and differing results. South Korea responded successfully, rapidly implementing key interventions for pandemic control, including widespread testing, early contact tracing, building health system capacity, and community education. ${ }^{9}$ Its implementation strategies reflected the nature of the epidemic (first wave and concentrated), a strong health system and public health capacity and capability, and receptive sociopolitical and community attitudes based on experience with SARS. For example, high rates of mobile phone coverage and technology to track individuals facilitated contact tracing that would not be possible in countries where culture and laws focus on data privacy.

The UK implemented some of the same interventions but had key contextual differences, including no recent experience of a similar pandemic and the nature of the outbreak at the time strategies were implemented (later start of the first wave and delayed initial response resulting in more diffuse spread). Furthermore, factors that facilitated the response in South Korea were challenges in the UK, where rapid coherent political engagement and action were lacking and the population was initially less responsive to public health messages, and some first cases were unrecognised.

Despite reporting its first cases after responses in South Korea and other countries were under way, the UK delayed in choosing implementation strategies (including for community education, strict social distancing, and testing), although many were effectively implemented once started. Contact tracing was initially planned, then abandoned in early March owing to lack of capacity. The UK moved from containment to a delay phase, and a policy of widespread contact tracing was not announced until mid-May. 
As we face the spread of covid-19 to new settings, including low income countries with differing resources and contexts, prepare for multiple waves, and try to reduce harms related to disruption of critical care, we need an implementation science approach more than ever. Public health efforts to stop the pandemic must be sustained without neglecting other evidence based interventions to maintain demand, access, and availability of other health services, including those for maternal and child health and chronic diseases. ${ }^{10}$ Recognising contextual factors and using appropriate implementation strategies ${ }^{11}$ can help ensure that lessons are learnt and evidence based responses are applied and spread faster than the pandemic itself, with sufficient adaptation to improve population health worldwide.

Competing interests: The BMJ has judged that there are no disqualifying financial ties to commercial companies. The authors declare no other interests. The BMJ policy on financial interests is here: www.bmj.com/sites/default/files/attachments/ resources/2016/03/16-current-bmj-education-coi-form.pdf.

Provenance and peer review: Not commissioned; externally peer reviewed.

1 Hwang S, Birken SA, Melvin CL, Rohweder CL, Smith JD. Designs and methods for implementation research: advancing the mission of the CTSA program. J Clin Trans/ Sci 2020. [Epub ahead of print.] 10.1017/cts.2020.16
2 Smith JD, Hasan M. Quantitative approaches for the evaluation of implementation research studies. Psychiatry Res 2020;283:112521-9. 10.1016/.jpsychres.2019.112521 31473029 3 Powell BJ, Waltz TJ, Chinman MJ, etal . A refined compilation of implementation strategies: results from the Expert Recommendations for Implementing Change (ERIC) project. Implement Sci 2015;10:21. 10.1186/s13012-015-0209-1 25889199

4 Elston JWT, Cartwright C, Ndumbi P, Wright J. The health impact of the 2014-15 Ebola outbreak. Public Health 2017;143:60-70. 10.1016/j.puhe.2016.10.020 28159028

5 Shultz JM, Cooper JL, Baingana F, etal . The role of fear-related behaviors in the 2013-2016 west Africa Ebola virus disease outbreak. Curr Psychiatry Rep 2016;18:104. 10.1007/s11920-016-0741-y 27739026

6 Helfrich CD, Weiner BJ, McKinney MM, Minasian L. Determinants of implementation effectiveness: adapting a framework for complex innovations. Med Care Res Rev 2007;64:279-303. 10.1177/1077558707299887 17507459

7 Damschroder LJ, Aron DC, Keith RE, Kirsh SR, Alexander JA, Lowery JC. Fostering implementation of health services research findings into practice: a consolidated framework for advancing implementation science. Implement Sci 2009;4:50. 10.1186/1748-5908-4-50 19664226

8 Aarons GA, Sklar M, Mustanski B, Benbow N, Brown CH. "Scaling-out" evidence-based interventions to new populations or new health care delivery systems. Implement $\mathrm{Sc}$ 2017:12:111. 10.1186/s13012-017-0640-6 28877746

9 Oh J, Lee J-K, Schwarz D, Ratcliffe HL, Markuns JF, Hirschhorn LR. National Response to covid-19 in the Republic of Korea and lessons learned for other countries. Health Syst Reform 2020;6:e1753464. 10.1080/23288604.2020.1753464 32347772

10 Roberton T, Carter ED, Chou VB, etal. Early estimates of the indirect effects of the coronavirus pandemic on maternal and child mortality in low- and middle-income countries. SSRN 2020.[Preprint] https://ssrn.com/abstract=3576549

11 Smith JD, Li D, Rafferty MR. The implementation research logic model: a method for planning, executing, reporting, and synthesizing implementation projects. medRxiv 2020.04.05.20054379. [Preprint]. 10.1101/2020.04.05.20054379

Published by the BMJ Publishing Group Limited. For permission to use (where not already granted under a licence) please go to http://group.bmj.com/group/rights-licensing/ permissions 